\title{
Erratum to: Isolation and analysis of differentially expressed genes during asexual sporulation in liquid static culture of Ganoderma lucidum by suppression subtractive hybridization
}

Jun-Wei Xu $\cdot$ Wei Zhao $\cdot$ Yi-Ning Xu • Jian-Jiang Zhong

Published online: 28 December 2011

(C) Springer Science+Business Media B.V. 2011

\section{Erratum to: Mol Biol Rep}

DOI 10.1007/s11033-011-1134-2

The original version of this article, unfortunately, contained a mistake.
The corrected corresponding author's affiliations are listed in this erratum.

The online version of the original article can be found under doi: 10.1007/s11033-011-1134-2.

J.-W. Xu · J.-J. Zhong ( $ه)$

State Key Laboratory of Bioreactor Engineering,

School of Biotechnology, East China University of Science and Technology, 130 Meilong Road, Shanghai 200237, China

e-mail: jjzhong@sjtu.edu.cn

J.-W. Xu · W. Zhao · Y.-N. Xu · J.-J. Zhong

Key Laboratory of Microbial Metabolism (Ministry

of Education), School of Life Sciences and Biotechnology,

Shanghai Jiao Tong University, 800 Dong-Chuan Road,

Shanghai 200240, China 